\title{
SLC26A6-selective inhibitor identified in a small-molecule screen blocks fluid absorption in small intestine
}

\author{
Onur Cil,, ${ }^{1}$ Peter M. Haggie, ${ }^{2}$ Joseph-Anthony Tapia Tan, ${ }^{2}$ Amber A. Rivera, ${ }^{2}$ and Alan S. Verkman ${ }^{2}$ \\ 'Department of Pediatrics and 'Departments of Medicine and Physiology, University of California, San Francisco, \\ San Francisco, California, USA.
}

\begin{abstract}
SLC26A6 (also known as putative anion transporter 1 [PAT1]) is a $\mathrm{Cl}^{-} / \mathrm{HCO}_{3}{ }^{-}$exchanger expressed at the luminal membrane of enterocytes where it facilitates intestinal $\mathrm{Cl}^{-}$and fluid absorption. Here, high-throughput screening of $\mathbf{5 0 , 0 0 0}$ synthetic small molecules in cells expressing PAT1 and a halide-sensing fluorescent protein identified several classes of inhibitors. The most potent compound, the pyrazolo-pyrido-pyrimidinone PAT1 ${ }_{\text {inh }}$-B01, fully inhibited PAT1-mediated anion exchange $\left(\mathrm{IC}_{50} \sim 350 \mathrm{nM}\right.$ ), without inhibition of the related intestinal transporter SLC26A3 (also known as DRA). In closed midjejunal loops in mice, PAT1 ${ }_{\text {inh }}$-B01 inhibited fluid absorption by $50 \%$,

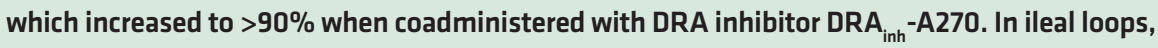
$\mathrm{PAT1}_{\text {inh }}$-B01 blocked fluid absorption by $>80 \%$, whereas $D R A_{\text {inh }}-A 270$ was without effect. In colonic loops, PAT1 ${ }_{\text {inh }}$-B01 was without effect, whereas DRA inh $-A 270$ completely blocked fluid absorption. In a loperamide constipation model, coadministration of PAT1 ${ }_{\text {inh }}-B 01$ with DRA ${ }_{\text {inh }}-\mathrm{A}_{270}$ increased stool output compared with DRA $\mathrm{inh}_{\mathrm{inh}}-\mathrm{A} 270$ alone. These results provide functional evidence for complementary and region-specific roles of PAT1 and DRA in intestinal fluid absorption, with PAT1 as the predominant anion exchanger in mouse ileum. We believe that PAT1 ${ }_{\text {inh }}-\mathrm{B} 01$ is a novel tool to study intestinal ion and fluid transport and perhaps a drug candidate for small intestinal hyposecretory disorders such as cystic fibrosis-related meconium ileus and distal intestinal obstruction syndrome.
\end{abstract}

Conflict of interest: The authors have declared that no conflict of interest exists.

Copyright: (c) 2021, Cil et al. This is an open access article published under the terms of the Creative Commons Attribution 4.0 International License.

Submitted: January 13, 2021

Accepted: April 21, 2021

Published: June 8, 2021

Reference information: JCl Insight. 2021;6(11):e147699.

https://doi.org/10.1172/jci.

insight.147699.

\section{Introduction}

Electroneutral $\mathrm{NaCl}$ absorption in the small intestine, which drives fluid absorption, is mediated by the parallel actions of $\mathrm{Na}^{+} / \mathrm{H}^{+}$and $\mathrm{Cl}^{-} / \mathrm{HCO}_{3}^{-}$exchangers. NHE3 is the dominant $\mathrm{Na}^{+} / \mathrm{H}^{+}$exchanger in small intestine (1), and the NHE3 inhibitor tenapanor was recently approved for treatment of irritable bowel syndrome with constipation (2). SLC26A3 (also known as downregulated in adenoma [DRA]) and SLC26A6 (also known as putative anion transporter 1 [PAT1]) are the principal anion exchangers at the luminal plasma membrane of intestinal epithelial cells (3). Due to the lack of selective inhibitors, current understanding of the functional roles of PAT1 and DRA in intestinal fluid transport is mainly from studies in knockout mice. Some earlier studies suggested PAT1 as the predominant anion exchanger in small intestine $(4,5)$, while another study reported DRA expression in small intestine and loss of small intestinal $\mathrm{Cl}^{-}$absorption in DRA-knockout mice (6). This difference may be the consequence of a combination of factors, including the lack of selective inhibitors, confounding effects of compensatory mechanisms in knockout mice, and possible species/strain differences.

A recent small-molecule screen, with follow-up compound optimization by medicinal chemistry, identified selective DRA inhibitors with nanomolar potency that blocked intestinal fluid absorption in mouse colon and were effective in experimental mouse models of constipation $(7,8)$. Here, to clarify the functional role of PAT1 in intestine, we identified and characterized a small-molecule PAT1-selective inhibitor, investigating its effects on intestinal fluid absorption in intestinal closed loops and testing its efficacy in a murine model of constipation, including additive action with a DRA inhibitor.

\section{Results}

PAT1 inhibitors identified by high-throughput screening. A small-molecule screen was developed to identify PAT1 inhibitors utilizing transfected FRT cells expressing PAT1 (murine slc26a6) together with a halide-sensing 
cytoplasmic YFP (Figure 1A). Adherent cells in a 96-well format were subjected to an inward gradient of $\mathrm{I}^{-}$to drive exchange of $\mathrm{I}^{-}$for $\mathrm{Cl}^{-}$and consequent quenching of the YFP fluorescence signal. Little fluorescence quenching was seen in nontransfected FRT cells in response to extracellular addition of $\mathrm{I}^{-}$(Figure 1B). In PAT1-expressing cells, there was a large and rapid decrease in fluorescence in the absence of test compound or with an inactive test compound. A reduced rate of fluorescence quenching was seen with an active compound or with a high concentration of the nonselective anion transporter inhibitor niflumic acid.

Screening of 50,000 chemically diverse drug-like small molecules produced 4 compounds that at 25 $\mu \mathrm{M}$ inhibited PAT1-mediated $\mathrm{Cl}^{-} / \mathrm{I}^{-}$exchange by more than $75 \%$ (Figure $1 \mathrm{C}$ ). The most potent compound, $\mathrm{PAT}_{\mathrm{inh}}$-B01, inhibited PAT1 in a concentration-dependent manner with $\mathrm{IC}_{50}$ of $350 \mathrm{nM}$ (Figure 1D), substantially better than that of $200 \mu \mathrm{M}$ for niflumic acid. PAT1-mediated anion exchange was electroneutral in these studies, as shown by absence of inhibitor effect on short-circuit current in the PAT1-expressing FRT cells (data not shown) under conditions used previously (9) that produced large signals with anion channels CFTR and TMEM16A.

In addition to testing the 50,000 synthetic small molecules with diverse chemical structures, 699 additional compounds and their chemical analogs were screened that were found previously $(7,10,11)$ to inhibit the related SLC26A anion exchangers DRA or pendrin. None of the DRA or pendrin inhibitor analogs inhibited PAT1-mediated $\mathrm{Cl}^{-} / \mathrm{I}^{-}$exchange by more than $20 \%$ at $25 \mu \mathrm{M}$. Limited structure-activity relationship studies were done on $\mathrm{PAT} 1_{\mathrm{inh}}$-B01 using commercially available pyrazolo-pyrido-pyrimidinone analogs. Of 269 analogs tested, 5 compounds showed PAT1 inhibition, albeit they were much weaker compared with PAT $1_{\mathrm{inh}}$-B01. Further characterization and in vivo studies were done with PAT $1_{\mathrm{inh}}$-B01 because of its better inhibition potency, compared with the other active compounds in Figure $1 \mathrm{C}$, and its favorable solubility and drug-like chemical structure.

Characterization of $P A T 1_{i n h}-B 01$ inhibition of PAT1 anion exchange. As PAT1 functions as a general monovalent anion exchanger, PAT1 ${ }_{\text {inh }}$-B01 inhibition was studied for different modes of anion exchange. PAT $1_{\text {inh }}{ }^{-}$ B01 inhibited PAT1-mediated $\mathrm{Cl}^{-} / \mathrm{SCN}^{-}$exchange with $\mathrm{IC}_{50}$ of $260 \mathrm{nM}$ (Figure 2A). To measure $\mathrm{Cl}^{-} /$ $\mathrm{HCO}_{3}^{-}$exchange, the time course of cytoplasmic $\mathrm{pH}$ was measured, using BCECF as a $\mathrm{pH}$ indicator, in response to a gluconate gradient to drive exchange of cytoplasmic $\mathrm{Cl}^{-}$with extracellular $\mathrm{HCO}_{3}^{-}$producing cytoplasmic alkalinization (Figure $2 \mathrm{~B}$ ) as done before for DRA and pendrin $(7,10,11)$. PAT $1_{\text {inh }}$-B01 inhibited $\mathrm{Cl}^{-} / \mathrm{HCO}_{3}^{-}$exchange with $\mathrm{IC}_{50}$ of $290 \mathrm{nM}$. Figure $2 \mathrm{C}$ shows the time course of inhibition of PAT1-mediated $\mathrm{Cl}^{-} / \mathrm{I}^{-}$exchange following addition of $1 \mu \mathrm{M}$ PAT1 ${ }_{\mathrm{inh}}-\mathrm{B} 01$. PAT1 inhibition increased progressively with time, with 50\% inhibition seen at approximately 3 minutes, suggesting an intracellular site of PAT $1_{\text {inh }}$-B01 action. Inhibition was fully reversible following PAT $1_{\text {inh }}$-B01 washout (data not shown).

An earlier study in intestinal Caco-2 and T84 cells suggested that PAT1 activity might be subject to regulation by secondary messengers such as cAMP (12). To test whether PAT1 is subject to direct regulation by cAMP, cGMP, or $\mathrm{Ca}^{2+}$, PAT1-mediated $\mathrm{Cl}^{-} / \mathrm{I}^{-}$exchange was measured in the PAT1-expressing FRT cells following incubations with forskolin plus IBMX, 8-Br-cGMP, or ATP. These agonists did not significantly affect PAT1-mediated $\mathrm{Cl}^{-} / \mathrm{I}^{-}$exchange (Figure 2D).

$P A T 1_{\text {inh }}{ }^{-B 01}$ selectivity. PAT1 ${ }_{\text {inh }}$-B01 selectivity was studied by testing its effects on related SLC26A family anion transporters and the $\mathrm{Cl}^{-}$channel TMEM16A. These measurements used a similar $\mathrm{Cl}^{-} / \mathrm{I}^{-}$exchange protocol, but FRT cells separately expressed each of the SLC26A family members or TMEM16A. As seen in original fluorescence quenching curves in Figure $3 \mathrm{~A}$ and as summarized in Figure $3 \mathrm{~B}, \mathrm{PAT} 1_{\text {inh }}$-B01 at a high concentration of $25 \mu \mathrm{M}$ did not significantly inhibit slc26a3, slc26a4, SLC26A9, or TMEM16A. PAT1 ${ }_{\text {inh }}$-B01 also did not affect the activities of slc26a1 and slc26a2 (Supplemental Figure 1; supplemental material available online with this article; https://doi.org/10.1172/jci.insight.147699DS1), other members of SLC26A family expressed in the intestine. PAT $1_{\text {inh }}$-B01 selectivity was also tested by short-circuit current $\left(\mathrm{I}_{\mathrm{sc}}\right)$ in well-differentiated human bronchial epithelial (HBE) cells, which have a complex ion transport mechanism that includes cystic fibrosis transmembrane conductance regulator (CFTR), epithelial $\mathrm{Na}^{+}$channel (ENaC), and $\mathrm{Ca}^{+2}$-activated chloride channels (CaCCs) (13). PAT1 ${ }_{\text {inh }}$-B01 pretreatment had no significant effect on $\mathrm{ENaC}, \mathrm{CFTR}$, or CaCC activities, as evidenced by comparable $\mathrm{I}_{\mathrm{sc}}$ responses to amiloride, forskolin/CFTR $\mathrm{inh}_{\mathrm{i}}-172$, and ATP, respectively (Figure 3C). Potential PAT1 ${ }_{\text {inh }}$-B01 cytotoxicity was studied in FRT cells. Prolonged (48 h) PAT1 ${ }_{\text {inh }}$-B01 incubation at a concentration of $10 \mu \mathrm{M}$ did not cause cytotoxicity, as measured by the Alamar Blue assay (Figure 3D).

$P A T 1_{\text {inh }}$-B01 blocks fluid absorption partially in mouse jejunum and completely in mouse ileum. Closed intestinal loop studies in mice were done using the selective inhibitors PAT $1_{\mathrm{inh}}-\mathrm{B} 01$ and $\mathrm{DRA}_{\mathrm{inh}}-\mathrm{A} 270$, individually and together, to investigate the contributions of PAT 1 and DRA in intestinal fluid absorption. PAT $1_{\text {inh }}$-B01 
A

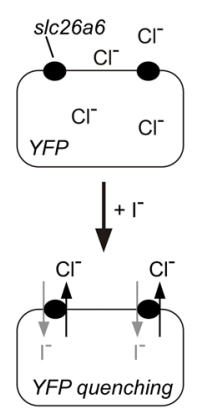

B

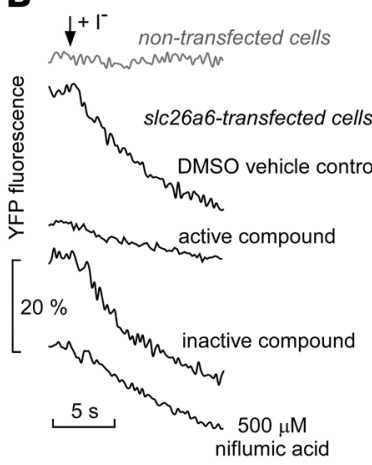

C

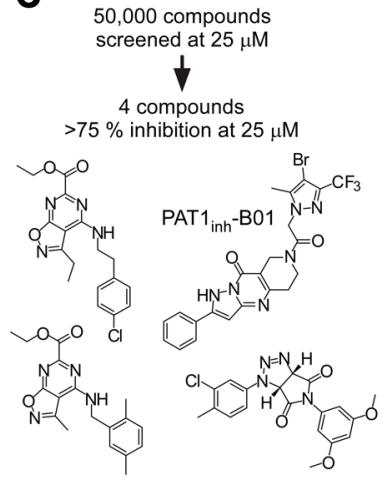

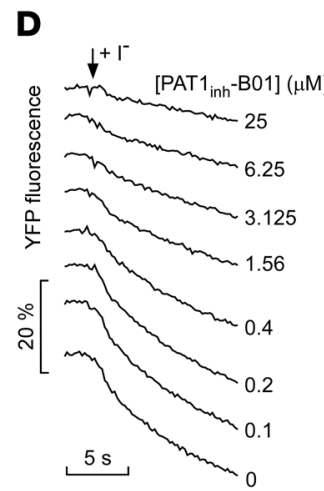

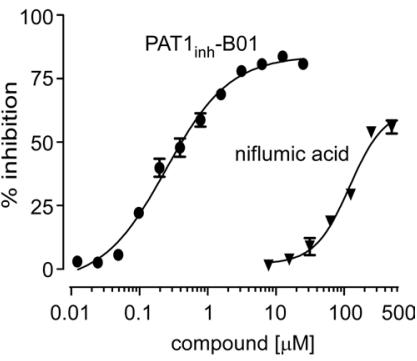

Figure 1. Small-molecule screen identifies PAT1 inhibitors. (A) Screening assay in which FRT cells coexpressing slc26a6 (PAT1) and a halide-sensing yellow fluorescent protein (YFP) were subject to an inwardly directed iodide $\left(\mathrm{I}^{-}\right)$gradient. PAT1-mediated influx of $\mathrm{I}^{-}$in exchange for $\mathrm{Cl}^{-}$reduces cytoplasmic $\mathrm{YFP}$ fluorescence. (B) Representative primary screening data from single wells of 96-well plates. Nal-containing solution (100 $\mu$ l) was injected onto cells bathed in $100 \mu \mathrm{l}$ of a NaClcontaining solution. YFP fluorescence curves shown for nontransfected cells (top curve) and for PAT1-expressing cells (bottom 4 curves). Cells were preincubated with vehicle (DMSO) control, test compound (examples of active and inactive compounds shown), or niflumic acid (positive control). (C) Summary of screening results (top), with chemical structures of active compounds identified in the primary screen (bottom). (D) Representative YFP fluorescence curves (left) and concentration dependence (right) for inhibition of PAT1-mediated $\mathrm{Cl}^{-} / \mathrm{I}^{-}$exchange by PAT1 ${ }_{\text {inh }}$-B01 and niflumic acid (mean $\pm \mathrm{SEM}, n=4$ ).

at $30 \mu \mathrm{M}$ in the lumen of closed jejunal loops inhibited the decrease in loop weight-to-length ratio, a direct measure of fluid absorption, by approximately $50 \%$ (Figure $4 \mathrm{~A}$ ). In parallel studies, $\mathrm{DRA}_{\mathrm{inh}}-\mathrm{A} 270$ at 10 $\mu \mathrm{M}$ also inhibited the reduction in loop weight-to-length ratio by approximately $50 \%$. PAT $1_{\text {inh }}$-B01 and $\mathrm{DRA}_{\mathrm{inh}}-\mathrm{A} 270$, when administered together, produced $>90 \%$ inhibition, comparable to that produced by the NHE3 inhibitor tenapanor at $10 \mu \mathrm{M}$. These results provide pharmacological evidence for comparable contributions of PAT1 and DRA to fluid absorption in mouse jejunum.

In ileal loops PAT $1_{\text {inh }}$-B01 fully blocked fluid absorption, as seen from prevention of the reduction in loop weight-to-length ratio at 30 minutes, which was comparable to the tenapanor effect (Figure 4B). In contrast, $\mathrm{DRA}_{\text {inh }}-\mathrm{A} 270$ did not inhibit ileal fluid absorption, and PAT1 ${ }_{\text {inh }}-\mathrm{B} 01$ and $\mathrm{DRA}_{\mathrm{inh}}-\mathrm{A} 270$ together had comparable efficacy to that of $\mathrm{PAT} 1_{\mathrm{inh}}-\mathrm{B} 01$ alone. These results provide evidence that PAT1 is the main anion exchanger in the mouse ileum. To further characterize the effects of PAT1 inhibition, loop fluid pH was measured in ileal loops in the absence and presence of $\mathrm{PAT}_{\mathrm{inh}}$-B01. In control loops, luminal $\mathrm{pH}$ increased to greater than 8.0 at 30 minutes after injection of $\mathrm{PBS}\left(\mathrm{pH}\right.$ 7.4) (Supplemental Figure 2A), consistent with $\mathrm{Cl}^{-}$/ $\mathrm{HCO}_{3}^{-}$exchange. The $\mathrm{pH}$ increase was prevented in loops containing $\mathrm{PAT}_{\mathrm{inh}}-\mathrm{B} 01$. These results suggest that the in vivo effect of PAT1 ${ }_{\text {inh }}{ }^{-B} 01$ on ileal fluid absorption is due to inhibition of PAT1-mediated $\mathrm{Cl}^{-} / \mathrm{HCO}_{3}^{-}$ exchange. Although they probably do not have a significant contribution in fluid transport, sodium-dependent phosphate cotransporters, including NaPi-IIb, are highly expressed in mouse ileum (14). To investigate the potential contribution of phosphate transporters on the antiabsorptive effect of $\mathrm{PAT}_{\text {inh }}$-B01 seen here, closedloop experiments were done in ileum in the absence of luminal phosphate using HEPES-buffered saline. In this setting, PAT1 $1_{\mathrm{inh}}$-B01 effectively blocked fluid absorption (Supplemental Figure $2 \mathrm{~B}$ ).

In closed distal colonic loops PAT $1_{\text {inh }}$-B01 did not inhibit the reduction in loop weight-to-length ratio, whereas, as reported previously (7), DRA $\mathrm{inh}_{\text {inh }}$-A270 completely prevented fluid absorption, as was also seen with PAT1 $1_{\text {inh }}$-B01 and DRA inh $_{\text {inh }}$-A270 together (Figure 4C). As reported previously (7), the NHE3 inhibitor tenapanor did not inhibit fluid absorption from mouse colonic loops.

Cystic fibrosis $(\mathrm{CF})$ is associated with intestinal hyposecretory disorders, such as meconium ileus and distal intestinal obstructive syndrome (DIOS), which primarily affect the distal small intestine (ileum) (15). To test the potential utility of PAT1 inhibition in these conditions, closed-loop studies were done in ilea of CF mice (F508del homozygous). As found in wild-type mice, PAT $1_{\text {inh }}-\mathrm{B} 01$ and tenapanor completely blocked fluid absorption from ilea of CF mice, whereas DRA ${ }_{\text {inh }}-A 270$ had no effect (Figure 5). These results support the potential utility of PAT1 inhibitors for CF-related small intestinal disorders.

Efficacy of PAT1 ${ }_{i n h}-B 01$ and $D R A_{i n h}-A 270$ in a loperamide model of constipation. The efficacy of PAT1 $1_{\text {inh }}-\mathrm{B} 01$ was tested in loperamide-induced constipation model in mice (Figure 6A). Loperamide produces marked constipation in mice, as indicated by reduced weight, number of pellets, and water content for stool collected over 3 hours (Figure $6 \mathrm{~B})$. PAT1 ${ }_{\text {inh }}-\mathrm{B} 01(10 \mathrm{mg} / \mathrm{kg}$, oral) did not affect the stool parameters, whereas 

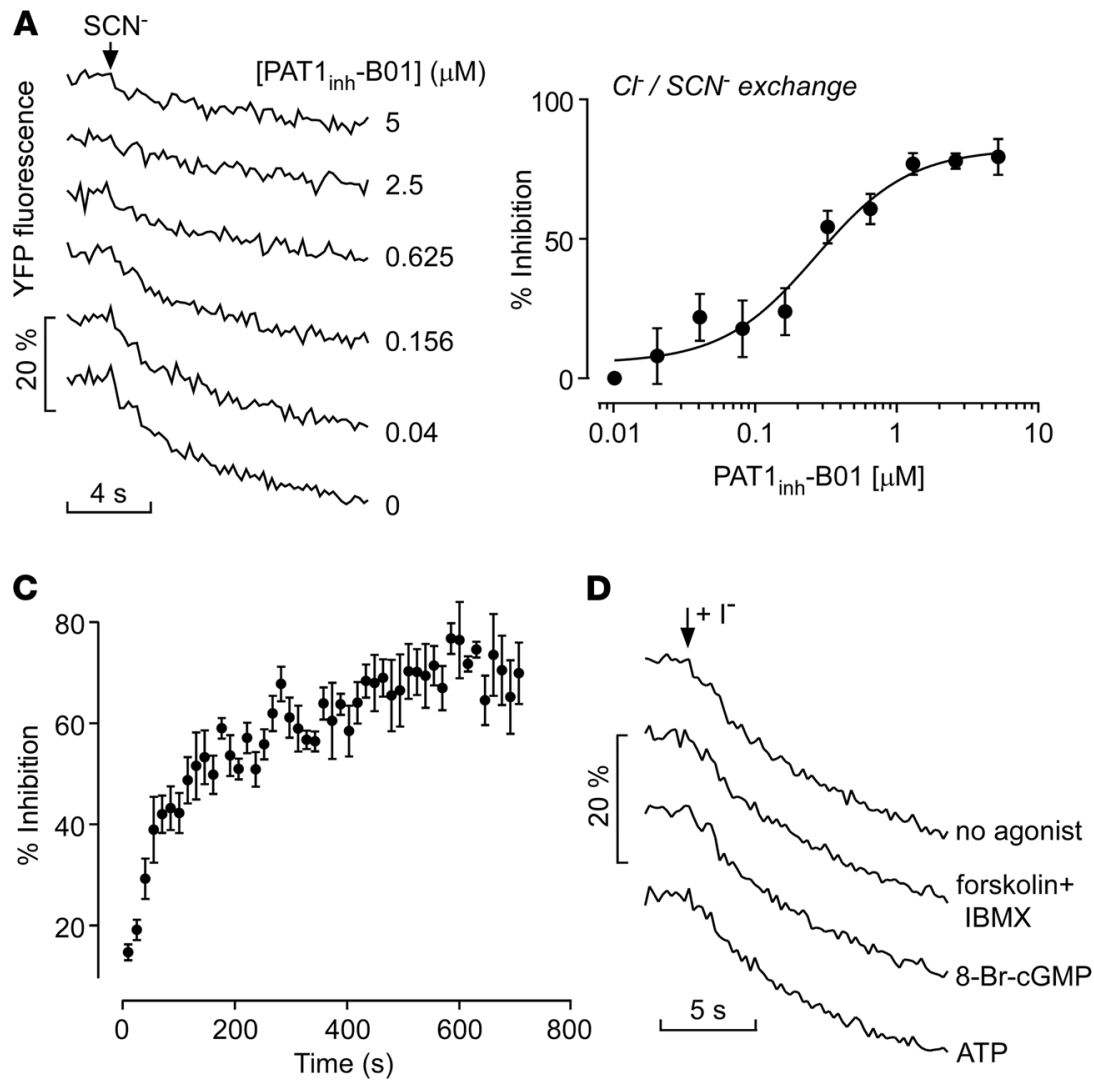

B
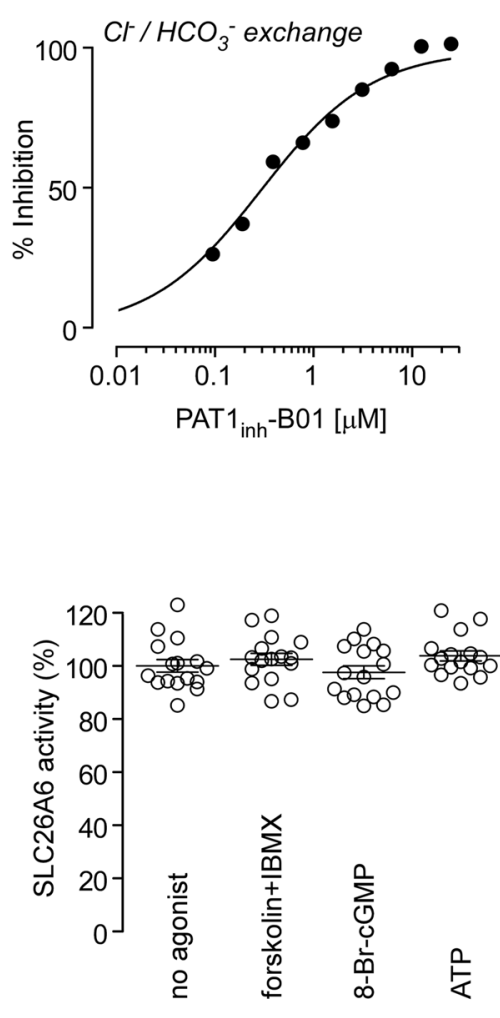

Figure 2. PAT1 ${ }_{\text {inh }}$-B01 inhibition of PAT1-mediated anion exchange. (A) YFP fluorescence quenching curves (left), as in Figure 1B, for inhibition of PAT1mediated exchange of $\mathrm{Cl}^{-}$for $\mathrm{SCN}^{-}$, with concentration dependence of $\mathrm{PAT1}_{\mathrm{inh}}{ }^{-} \mathrm{BO1}$ effect (right) (mean $\pm \mathrm{SEM}, n=3$ ). (B) Inhibition of $\mathrm{Cl}^{-} / \mathrm{HCO}_{3}{ }^{-}$exchange measured in BCECF-loaded FRT cells by PAT1 $1_{\text {inh }}$-B01 (mean \pm SEM, $n=16-32$ ). (C) Time course of PAT1 inhibition following addition of $1 \mu M$ PAT1 ${ }_{\text {inh }}$-B01 to cells (mean $\pm \mathrm{SEM}, n=3$ ). (D) Effects of second messenger signaling on PAT1-mediated $\mathrm{Cl}^{-} / \mathrm{I}^{-}$exchange. YFP fluorescence quenching curves (left) and summary data (right) following cell exposure to agonists of cAMP ( $20 \mu \mathrm{M}$ forskolin $+200 \mu \mathrm{M}$ IBMX), cGMP (100 $\mu \mathrm{M}$ 8-Br-cGMP) and Ca+2 (100 $\mu \mathrm{M}$ ATP), or vehicle control (1\% DMSO). Mean \pm SEM, $n=16$ experiments per group, differences not significant by 1-way ANOVA and post hoc Newman-Keuls multiple comparisons test.

$\mathrm{DRA}_{\text {inh }}-\mathrm{A} 270(10 \mathrm{mg} / \mathrm{kg}$, oral) normalized stool water content and increased stool weight and number of pellets, as reported previously (8). Coadministration of PAT1 ${ }_{\mathrm{inh}}-\mathrm{B} 01$ and $\mathrm{DRA}_{\mathrm{inh}}-\mathrm{A} 270$ increased stool output significantly more than $\mathrm{DRA}_{\text {inh }}$-A270 alone. PAT1 inhibition alone thus did not affect stool output in mice, whereas it potentiated the laxative action of DRA inhibition.

\section{Discussion}

A selective inhibitor of PAT1-mediated anion exchange was identified and characterized in order to investigate the relative contributions of PAT1 and DRA to fluid absorption in different regions of mouse intestine. Current understanding of the contributions of PAT1 and DRA in intestinal fluid absorption comes mainly from studies in knockout mice, as selective inhibitors have not been available. An early study showed strong PAT1 mRNA expression throughout the small intestine (duodenum, jejunum, and ileum) in mice, with no expression in the colon (5), whereas DRA mRNA was highly expressed in the colon with low-level expression throughout the small intestine. In mouse duodenum, Simpson et al. (4) reported comparable PAT1 and DRA mRNA expression, with $\mathrm{Cl}^{-} / \mathrm{HCO}_{3}{ }^{-}$exchange activity reduced by $65 \%-80 \%$ and $30 \%-40 \%$ in PAT1and DRA-knockout mice, respectively. Here, we did not investigate duodenal fluid absorption because the mouse duodenum is a short and fixed segment, precluding the generation of closed loops.

In jejunum, Walker et al. (6) reported that net $\mathrm{Cl}^{-}$absorption was reduced by more than $80 \%$ in DRAknockout mice but by less than $20 \%$ in PAT1-knockout mice. They concluded that DRA is the major apical anion exchanger in mouse jejunum, though found only modest DRA immunofluorescence. Another study reported approximately 50\% reduction in net $\mathrm{Cl}^{-}$absorption in jejunum in PAT1-knockout mice (16). The same group, using an in vivo perfusion technique, showed comparably reduced fluid absorption by approximately $30 \%$ in jejunum of both DRA- and PAT1-knockout mice (17). The functional data here using 
A

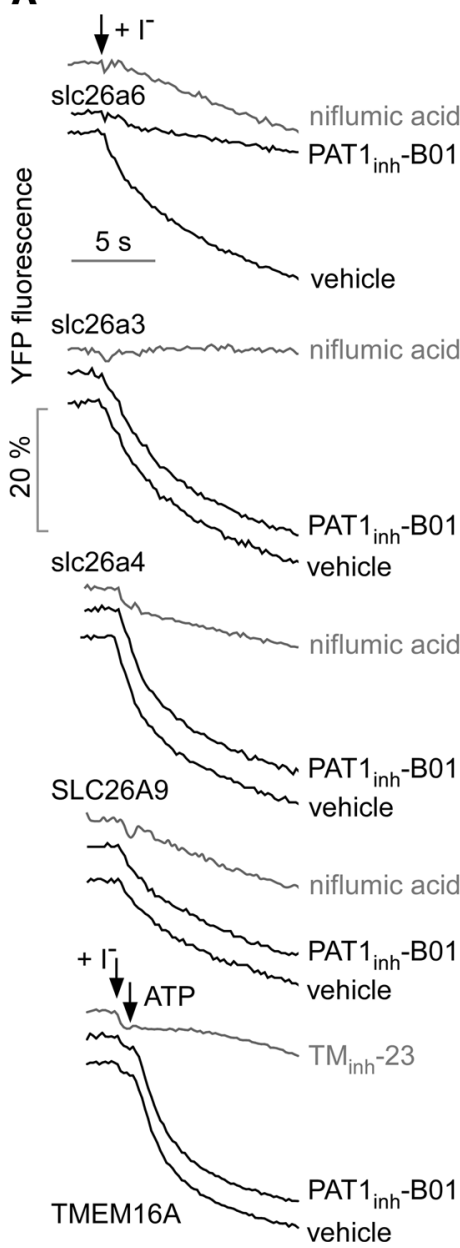

B

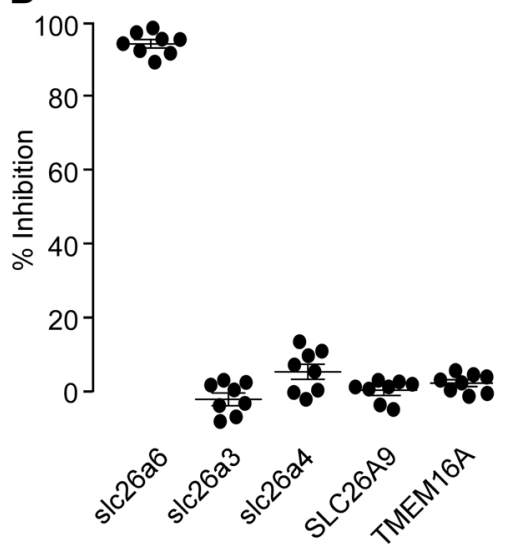

C

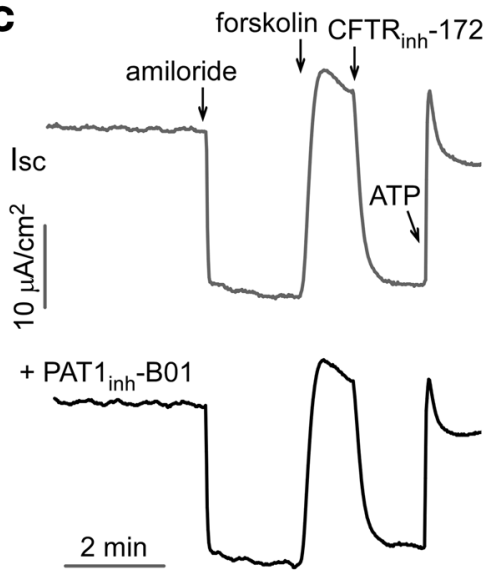

D

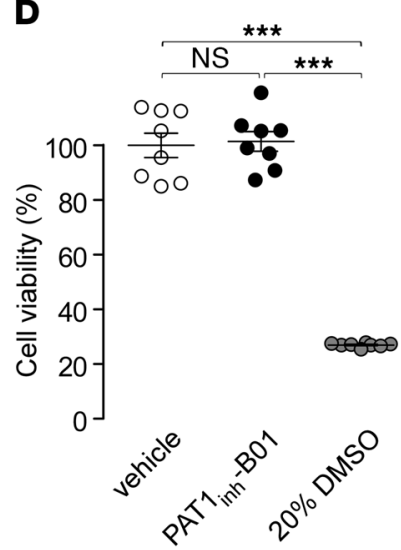

- vehicle
- PAT1 ${ }_{\text {inh }}$-B01

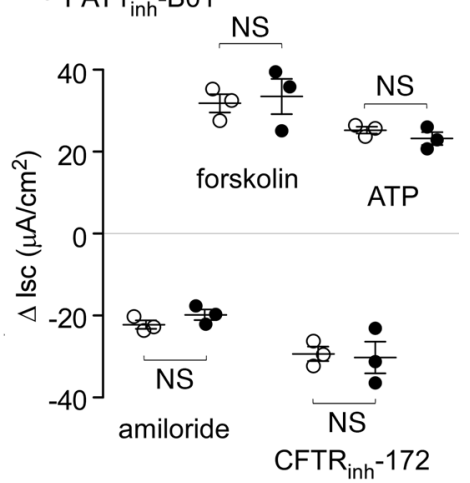

Figure 3. PAT1 ${ }_{\text {inh }}$-B01 selectivity and cytotoxicity. (A) Selectivity of PAT1 ${ }_{\text {inh }}-\mathrm{B} 01$ against related SLC26A family members and TMEM16A $\mathrm{Cl}^{-}$channel. $\mathrm{Cl}^{-} / \mathrm{I}^{-}$ exchange or l' influx measured in FRT cells coexpressing YFP and SLC26A isoforms or TMEM16A, with data shown for $25 \mu$ M PAT1 ${ }_{\text {inh }}{ }^{-B 01,}$ DMSO vehicle control, and $500 \mu \mathrm{M}$ niflumic acid (positive control for SLC26A transporters) or $10 \mu \mathrm{M} \mathrm{TM}$ inh -23 (positive control for TMEM16A). (B) Percentage inhibition of $\mathrm{Cl}^{-} / \mathrm{I}^{-}$exchange or $\mathrm{I}^{-}$influx from data in $\mathbf{A}$ (mean $\pm \mathrm{SEM}, n=8$ experiments per condition). (C) (left) Short-circuit current ( $\mathrm{I}_{\mathrm{sc}}$ ) in well-differentiated HBE cells

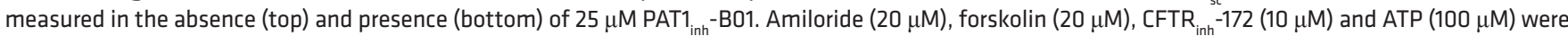
added as indicated. (right) Summary of $\mathrm{I}_{\mathrm{sc}}$ changes in the absence and presence of PAT1 ${ }_{\text {inh }}$-B01. Mean $\pm \mathrm{SEM}, n=3$, differences not significant by Student's $t$ test. (D) Cell viability assayed by Alamar blue in FRT cells incubated for 48 hours with vehicle control ( $0.1 \%$ DMSO) or $10 \mu M$ PAT $1_{\text {inh }}$-B01. $20 \%$ DMSO was used as positive control. Mean $\pm \mathrm{SEM}, n=8$ wells per group, 1-way ANOVA and post hoc Newman-Keuls multiple comparisons test, ${ }^{* * *} P<0.001$.

selective inhibitors are consistent with the latter studies and suggest comparable and complementary roles for PAT1 and DRA in jejunal fluid absorption in mice. We previously reported that the original, relatively low-affinity DRA inhibitor, $\mathrm{DRA}_{\mathrm{inh}}-\mathrm{A} 250$, did not significantly affect fluid absorption in closed midjejunal loops in mice at $10 \mu \mathrm{M}$ (7). In the current study, the 5-fold more potent DRA inhibitor DRA $\mathrm{inh}_{\text {inh }}$-A270 partially blocked fluid absorption in mouse jejunum at $10 \mu \mathrm{M}$, suggesting that DRA has a role in fluid absorption in this segment of the mouse intestine.

Whittamore et al. (18) investigated the relative contributions of PAT1 and DRA on ileal $\mathrm{Cl}^{-}$absorption using knockout mice in a C57BL/6 genetic background. They reported that PAT1 knockout had no effect on net absorptive $\mathrm{Cl}^{-}$flux across mouse ileum and that DRA knockout reduced absorptive $\mathrm{Cl}^{-}$flux by $40 \%$. The current study, using selective pharmacological inhibitors in CD1 mice, found that PAT1 is the main luminal anion exchanger responsible for fluid absorption in mouse ileum, with little contribution of DRA. This difference may be due to compensatory changes in knockout mice and/or strain differences (C57BL/6 vs. CD1). Further studies using selective PAT1 and DRA inhibitors in different animals and mouse strains may be informative. DRA is believed to be the major anion exchanger in mouse colon (3), as PAT1 mRNA is not expressed there (5). Consistent with these studies, PAT1 inhibition did not affect fluid absorption in mouse colonic loops under conditions in which $\mathrm{DRA}_{\text {inh }}$-A270 fully inhibited fluid absorption. 
A

A Jejunum

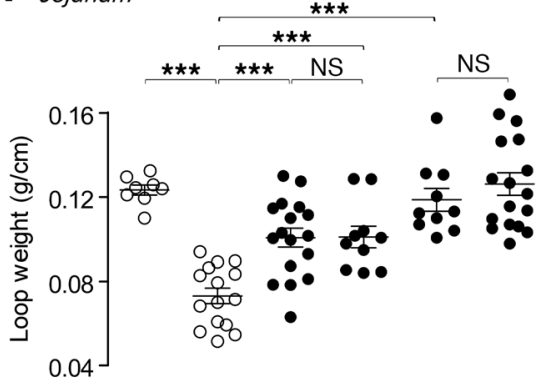

$\begin{array}{lllllll}\text { Time (min) } & 0 & 30 & 30 & 30 & 30 & 30\end{array}$

$\mathrm{PAT}_{\text {inh }}$-B01 - $-\quad+\quad-\quad+\quad-$

$\mathrm{DRA}_{\text {inh }}$-A270

tenapanor
B lleum
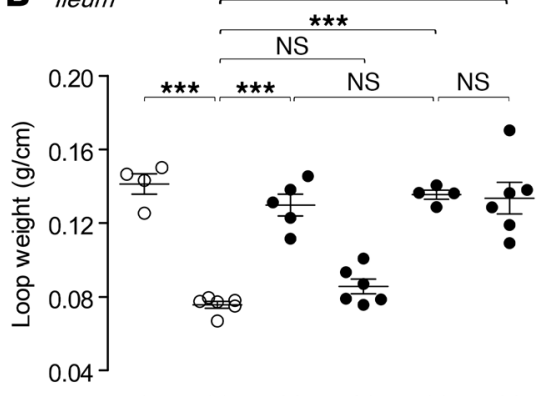

$\begin{array}{llllll}0 & 30 & 30 & 30 & 30 & 30\end{array}$
C Colon

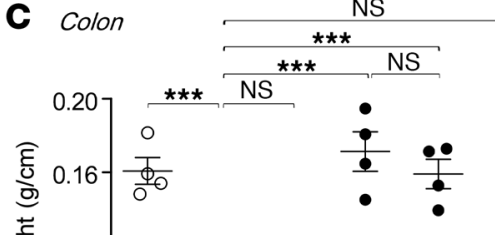

要

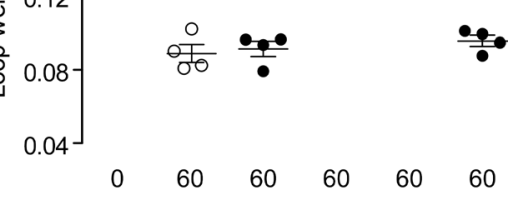

Figure 4. PAT1 inhibition blocks fluid absorption in mouse small intestine but not colon. (A) Effects of luminal PAT1 $1_{\text {inh }}-B 01$ (30 $\left.\mu M\right)$, DRA $\mu \mathrm{M})$, and tenapanor $(10 \mu \mathrm{M})$, individually and together, on loop weight-to-length ratio at 30 minutes in mouse midjejunal closed loops ( $n=8-17$ loops per group). (B) Effects of the compounds in mouse ileal closed loops ( $n=4-6$ loops per group). (C) Effects of the compounds in mouse distal colonic closed loops ( $n=4$ loops per group). Mean \pm SEM, 1-way ANOVA and post hoc Newman-Keuls multiple comparisons test, ${ }^{* * *} P<0.001$.

Loss of prosecretory CFTR activity is thought to be the principal cause of gastrointestinal disorders in CF, including chronic constipation, which has up to $47 \%$ prevalence, as well as the less common but more serious meconium ileus and DIOS $(15,19)$. We previously showed efficacy of DRA inhibition in a loperamide constipation model in wild-type and $\mathrm{CF}$ mice (7). Here, we found additive antiabsorptive effects of PAT $1_{\mathrm{inh}}-\mathrm{B} 01$ and $\mathrm{DRA}_{\mathrm{inh}}-\mathrm{A} 270$ in mouse jejunum, suggesting potential synergy of PAT1 and DRA inhibition for treatment of constipation. This is also supported by the increased stool output when PAT1 and DRA inhibitors were used together in a constipation model in mice. Meconium ileus and DIOS are caused by excessive dehydration of intestinal luminal contents primarily in ileum (15), where PAT1 has the predominant role in fluid absorption, as found here. There is limited information in the literature on PAT1 expression in CF mouse intestine. Early studies in CF mice focusing on duodenum reported varying results for PAT1 expression. Knauf et al. (20) showed that PAT1 expression is reduced by $40 \%$ in duodenum of CF mice compared with that in wild-type mice, whereas Simpson et al. (21) showed similar PAT1 mRNA expression in the duodena of CF and wildtype mice. Our closed loop studies suggest comparable PAT1 activity in wild-type and CF mouse ilea, based on similar reductions in loop weight-to-length ratio and similar efficacy of PAT1 ${ }_{\text {inh }}$-B01. The results here suggest the potential efficacy of PAT1 inhibitors for gastrointestinal conditions that affect small intestine. PAT1 inhibitors, by blocking fluid absorption in distal small intestine, are predicted to hydrate ileal luminal contents, which might prevent or treat obstruction in meconium ileus and DIOS.

We note that PAT1 is expressed in several organs outside of the intestine, including kidneys, heart, and pancreatic ducts, raising the issue of potential adverse effects such as arrhythmias (22); however, problems related to extraintestinal PAT1 expression have not been reported so far in humans with SLC26A6 mutations. Development of a nonabsorbable PAT1 inhibitor (similar to the NHE3 inhibitor tenapanor; ref. 2), may be useful in this regard. A concern with chronic PAT1 inhibition is the hyperoxaluria and calcium oxalate nephrolithiasis seen in PAT1-knockout mice due to impaired intestinal oxalate secretion (23); however, SLC26A6 variants in humans are not associated with hyperoxaluria or nephrolithiasis (24). Thus, it is unclear whether hyperoxaluria would be a side effect of chronic PAT1 inhibitor treatment in humans. These potential side effects would be of lesser concern for the short-term pharmacological PAT1 inhibition, as might be used for meconium ileus and DIOS.

Although classified as a solute carrier family, there is limited sequence similarity between various slc26a isoforms (25). In intestinal epithelial cells, 4 slc26a isoforms (slc26a1, slc26a2, slc26a3, and slc26a6) are mainly expressed, with apparently different expression levels in different segments (26). Here, we provide functional evidence that PAT $1_{\text {inh }}$-B01 does not affect ion transport mediated by other major intestinal slc26a isoforms, ion channels, or transporters. The observed in vivo effect of $\mathrm{PAT}_{\text {inh }}$ - $\mathrm{B} 01$ is therefore due to its selective action on PAT1.

With regard to the compound PAT1 ${ }_{\text {inh }}-\mathrm{B} 01$, there are no previous biological reports on the pyrazolopyrido-pyrimidinone scaffold, though a class of pyrimido-pyrazolo-quinolines that shares some structurally similarity was reported to restore chemosensitivity of colonic cancer cells in vitro (27). Pyrazolo-pyrido-pyrimidinone scaffolds can be prepared in 3-6 steps from commercially available starting chemicals. PAT1 ${ }_{\text {inh }}$-B01 has drug-like 


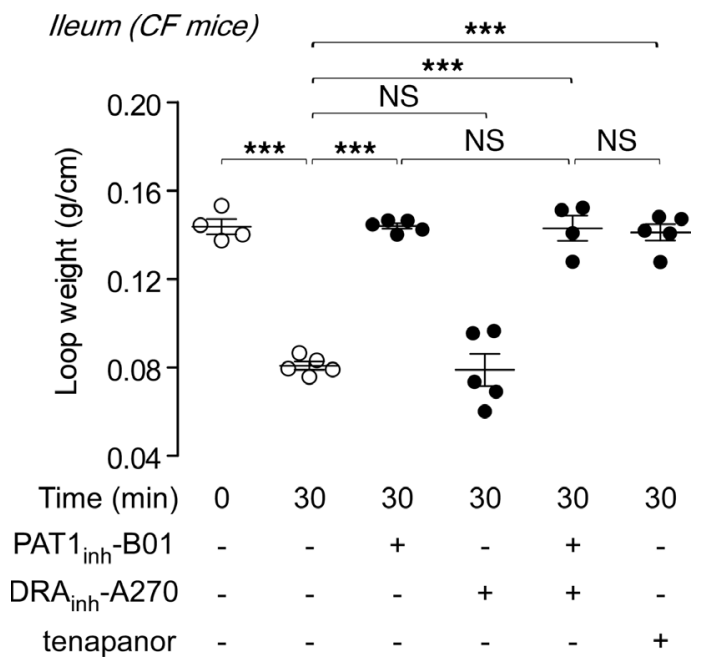

Figure 5. PAT1 inhibition blocks fluid absorption in ileum of cystic fibrosis mice. Effects of PAT1 $1_{\text {inh }}-B 01(30 \mu M), D R A_{\text {inh }}-A 270$ $(10 \mu \mathrm{M})$, and tenapanor $(10 \mu \mathrm{M})$, as studied in Figure 4, in ileal closed loops in cystic fibrosis (CF) mice (homozygous F508del, $n=4-6$ loops per group). Mean \pm SEM, 1-way ANOVA and post hoc Newman-Keuls multiple comparisons test, ${ }^{* * *} P<0.001$.

physiochemical properties, including the presence of multiple hydrogen bond acceptors, with partition coefficient (aLogP), molecular weight, and topological polar surface areas of $3.3,535$, and $88.3 \AA^{2}$, respectively. Further medicinal chemistry efforts may produce analogs with greater potency.

In conclusion, to our knowledge the PAT1-selective inhibitor identified herein is a novel research tool that was used to resolve the relative functional contributions of PAT1 and DRA on fluid absorption in different intestinal segments. PAT1 inhibitors should be useful for further physiological studies of intestinal $\mathrm{Cl}^{-}$and $\mathrm{HCO}_{3}^{-}$transport as well as PAT1 function in other organs where it is expressed. PAT1 inhibitors are also potential drug candidates for certain intestinal disorders, including CF-related meconium ileus and DIOS.

\section{Methods}

Chemicals. All chemicals were purchased from MilliporeSigma unless otherwise specified. DRA $\mathrm{inh}_{\mathrm{in}} \mathrm{A} 270$ was synthesized and purified as described previously (8).

cDNA constructs. A vector containing (murine) slc $26 \mathrm{a} 6 \mathrm{cDNA}$ was purchased from Origene (cDNA clone MC202861) and confirmed by sequence analysis. The slc26a6 cDNA was excised by digestion with SpeI and NotI and subcloned into the pLVX-IRES-mCherry lentiviral expression cassette (Clontech) at NheI and NotI sites. To generate a cell line for screening, slc26a6 lentiviral particles were produced in HEK293 cells using the pRSV-Rev, pMDLg/pRRE, and pMD2.G packaging vectors (Addgene, deposited by Didier Trono, plasmids 12253,12251 , and 12259) and used to transduce Fischer rat thyroid (FRT) cells previously transduced with lentivirus expressing EYFP-H148Q/I152L/F46L (YFP) (7). FRT cells were obtained from the University of California, San Francisco, Cystic Fibrosis Drug Discovery Core Center. For lentivirus production, HEK293T cells (ATCC, CRL-3216) were transfected with pLVX-IRES-mCherry-slc26a6, pRSVRev, $\mathrm{pMDLg} / \mathrm{pRRE}$, and pMD2.G using NanoFect transfection reagent (Alstem) per the manufacturer's instructions. After 1 day, the medium serum content was reduced to $2.5 \%$, and after 2 days, the medium was harvested, centrifuged (5 min, 5000g) and concentrated using Microcon centrifugal filters (MilliporeSigma) with 10-kDa molecular-weight cutoff.

Cell culture. Clonal FRT cell lines were generated by limited dilution, examined by fluorescence microscopy to confirm mCherry and YFP expression, and functionally characterized to confirm slc26a6 activity to generate the cell line FRT-YFP-slc26a6. FRT cell lines coexpressing YFP and slc26a3, slc26a4, SLC26A9, or TMEM16A were previously described $(7,11,28)$. FRT cells were cultured with Kaign's modified Ham's F12 medium supplemented containing 10\% FBS, $2 \mathrm{mM} \mathrm{L-glutamine,} 100 \mathrm{U} / \mathrm{ml}$ penicillin, $100 \mu \mathrm{g} / \mathrm{ml}$ streptomycin, $18 \mu \mathrm{g} / \mathrm{ml}$ myo-inositol, and $45 \mu \mathrm{g} / \mathrm{ml}$ ascorbic acid, with appropriate selection antibiotics. Well-differentiated HBE cells were cultured at an air-liquid interface on inserts as described previously (13). HBE cells were used for short-circuit current experiments 3 weeks after plating when they formed a tight epitheli$\operatorname{um}\left(R_{\mathrm{TE}}>1000 \Omega \mathrm{cm}^{2}\right)$. 
A

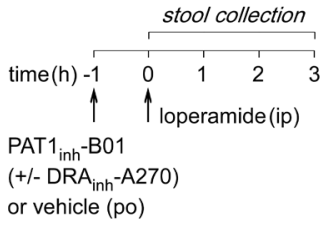

or vehicle $(1+10$
B

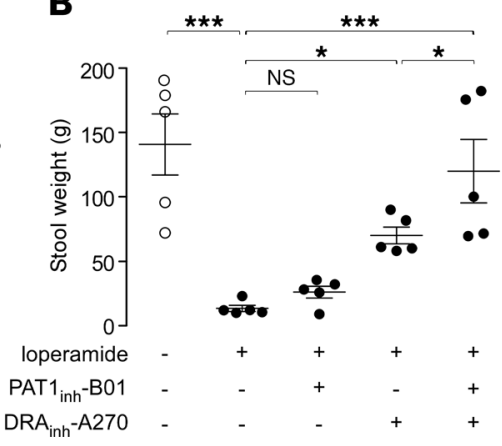

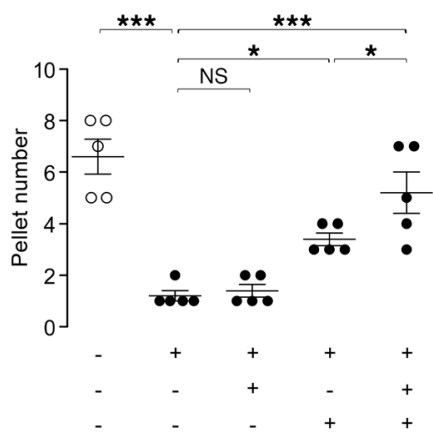

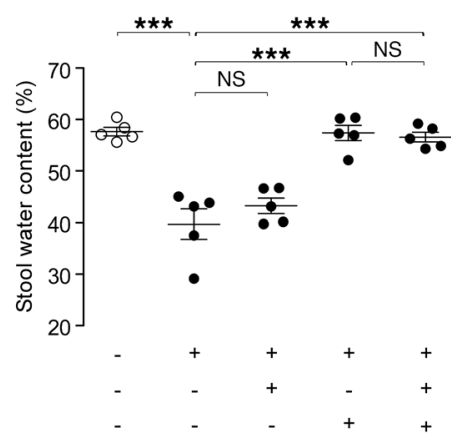

Figure 6. PAT1 and DRA inhibitors act in synergy in a loperamide model of constipation in mice. (A) Experimental protocol. (B) Effects of orally administered PAT1 1 -B01 $(10 \mathrm{mg} / \mathrm{kg})$ and DRA ${ }_{\text {inh }}-\mathrm{A} 270(10 \mathrm{mg} / \mathrm{kg})$, individually and together, on 3-hour stool weight (left), number of pellets (middle), and stool water content (right) in wild-type mice. Mean \pm SEM, $n=5$ mice per group, 1-way ANOVA and post hoc Newman-Keuls multiple comparisons test, ${ }^{*} P<0.05,{ }^{* * *} P<0.001$.

High-throughput screening. A collection of approximately 50,000 diverse, drug-like synthetic small molecules (ChemDiv) was screened at a concentration of $25 \mu \mathrm{M}$ using a Beckman Coulter BioMek FX liquid handling platform with FLUOstar OMEGA plate readers (BMG Labtech). FRT-YFP-slc26a6 cells were plated in 96-well black-walled, clear-bottom plates (Corning Life Sciences) at a density of 20,000 cells/well and cultured for 48 hours until confluent. Cells were washed twice in PBS and incubated for 10 minutes prior to assay in $100 \mu \mathrm{PBS}$ containing test compounds. For assay of slc26a6 function, baseline cellular fluorescence was measured for 2 seconds, after which $100 \mu \mathrm{NaI}$-substituted PBS $(140 \mathrm{mM} \mathrm{NaCl}$ replaced by $140 \mathrm{mM} \mathrm{NaI}$ ) was added by syringe pump to drive $\mathrm{Cl}^{-} / \mathrm{I}^{-}$exchange. The initial rate of $\mathrm{Cl}^{-} / \mathrm{I}^{-}$exchange was determined by single exponential regression of the fluorescence time course. All assay plates contained wells with negative (1\% DMSO) and positive (500 $\mu \mathrm{M}$ niflumic acid) controls. For structure-activity relationship studies, analogs of active compounds were purchased from ChemDiv.

Selectivity studies and additional assessment of slc26a6 activity. YFP-based cellular assays of slc26a3, slc26a4, SLC26A9, and TMEM16A function were performed as described previously (7). Slc26a1 and slc26a2 assays were done in transfected HEK 293 cells (see Supplemental Methods for details). Niflumic acid was used as a positive control for SLC26A isoforms (7). For TMEM16A, the selective small-molecule inhibitor $\mathrm{TM}_{\mathrm{inh}}-23$ was used as a positive control (28). The activities of $\mathrm{ENaC}, \mathrm{CFTR}$, and $\mathrm{CaCC}$ were determined using well-differentiated $\mathrm{HBE}$ cells as described previously (13). $\mathrm{Cl}^{-} / \mathrm{HCO}_{3}^{-}$exchange was measured as described previously $(7,10,11)$. Briefly, cell cytoplasm was labeled with the $\mathrm{pH}$-sensitive fluorescent dye BCECF-AM (Invitrogen), and $\mathrm{Cl}^{-} / \mathrm{HCO}_{3}^{-}$exchange was measured in response to addition of a gluconate-containing solution to drive exchange of cytoplasmic $\mathrm{Cl}^{-}$for extracellular $\mathrm{HCO}_{3}^{-}$, producing cytoplasmic alkalinization.

Cellular toxicity. FRT cells were plated in black-walled, clear-bottom tissue culture plates at a density of 20,000 cells/well. After 24 hours in culture, cells were incubated with $10 \mu \mathrm{M}$ PAT ${ }_{\text {inh }}$-B01, $0.1 \%$ DMSO (vehicle control), or $20 \%$ DMSO (positive control) for 48 hours for assay of cell viability using Alamar Blue (Thermo Fischer Scientific) according to the manufacturer's instructions.

Animals. Wild-type CD1 mice (male and female, age 10-16 weeks) and CF mice (homozygous CFTR F508del, male and female, age 12-20 weeks) were bred in the University of California, San Francisco, Laboratory Animal Resource Center. Animals were housed in communal cages with standard rodent chow and water available ad libitum.

Closed-loop studies of intestinal fluid absorption. For fluid absorption studies in small intestine, mice were given free access to $5 \%$ dextrose in water but not solid food for 24 hours before experiments. Closed midjejunal and ileal loops were prepared and isolated as described previously (29). Mice were anesthetized with isoflurane, and body temperature was maintained during surgery at $36^{\circ} \mathrm{C}-38^{\circ} \mathrm{C}$ using a heating pad. A small abdominal incision was made to expose small intestine for isolation of 3-4 closed small intestinal loops (jejunum or ileum, length 2-3 cm) with sutures. Loops were injected with $100 \mu \mathrm{L}$ PBS (pH 7.4, $137 \mathrm{mM} \mathrm{NaCl}, 2.7 \mathrm{mM} \mathrm{KCl}, 8 \mathrm{mM} \mathrm{Na}_{2} \mathrm{HPO}_{4}, 1.8 \mathrm{mM} \mathrm{KH}_{2} \mathrm{PO}_{4}, 1 \mathrm{mM} \mathrm{CaCl}, 0.5 \mathrm{mM} \mathrm{MgCl}$ ) containing $30 \mu \mathrm{M}$ PAT1 ${ }_{\text {inh }}-\mathrm{B} 01,10 \mu \mathrm{M} \mathrm{DRA}_{\text {inh }}-\mathrm{A} 270$, or $10 \mu \mathrm{M}$ tenapanor (MedChemExpress) (or vehicle, $0.1 \%$ DMSO in PBS). In some experiments, $30 \mu \mathrm{M} \mathrm{PAT} 1_{\text {inh }}-\mathrm{B} 01$ and $10 \mu \mathrm{M} \mathrm{DRA}_{\text {inh }}-\mathrm{A} 270$ were used together. In some experiments to rule out effects of sodium-dependent phosphate transport, the loop lumen contained HEPES-buffered saline ( $\mathrm{pH} 7.4,137 \mathrm{mM} \mathrm{NaCl}, 4.5 \mathrm{mM} \mathrm{KCl}, 10 \mathrm{mM}$ HEPES, $1 \mathrm{mM} \mathrm{CaCl}$, $0.5 \mathrm{mM}$ 
$\mathrm{MgCl}_{2}$ ). The abdominal incision was closed with sutures, and mice were allowed to recover from anesthesia. Intestinal loops were removed at 0 and 30 minutes (in separate mice), and loop length and weight were measured to quantify fluid absorption. In parallel experiments, CF mice were used and loop studies were done as described above. In some experiments, loop fluid was emptied with a syringe, and $\mathrm{pH}$ was measured immediately using $\mathrm{AB} 15 \mathrm{pH}$ meter after brief centrifugation at $2000 \mathrm{~g}$ for 5 minutes at $4^{\circ} \mathrm{C}$.

For fluid absorption studies in colon, mice were given free access to $5 \%$ dextrose in water but not solid food for 48 hours. Mice were treated with $500 \mu \mathrm{L}$ mineral oil twice a day rectally (final dose $16 \mathrm{~h}$ before surgery) during this period to evacuate the colon. Closed distal colonic loops (length $1.5-2 \mathrm{~cm}, 1$ loop per mouse) were isolated as described previously (7). Loops were injected with $100 \mu \mathrm{L}$ PBS with $30 \mu \mathrm{M}$ PAT$1_{\text {inh }}$-B01, $10 \mu \mathrm{M} \mathrm{DRA}_{\text {inh }}$-A270, or $10 \mu \mathrm{M}$ tenapanor (or vehicle). In some experiments, $30 \mu \mathrm{M} \mathrm{PAT} 1_{\text {inh }}$-B01 and $10 \mu \mathrm{M} \mathrm{DRA}_{\mathrm{inh}}-\mathrm{A} 270$ were used together. Colonic loops were removed at 0 and 60 minutes (in separate mice), and loop length and weight were measured to quantify fluid absorption.

Loperamide model of constipation. Wild-type CD1 mice were administered loperamide $(0.3 \mathrm{mg} / \mathrm{kg}$, intraperitoneally) in PBS containing $5 \%$ ethanol $(0.1 \mathrm{mg} / \mathrm{mL}$ final concentration) to induce constipation $(9,30)$. $\mathrm{PAT}_{\text {inh }}$-B01 and DRA ${ }_{\text {inh }}$-A270, individually or together $(10 \mathrm{mg} / \mathrm{kg}$ each, in saline containing 5\% DMSO and $10 \%$ Kolliphor HS 15), or vehicle control were administered by oral gavage 1 hour before loperamide. After loperamide injection mice were placed individually in metabolic cages with free access to food and water. Stool samples were collected for 3 hours, and total stool weight and number of fecal pellets were quantified. To measure stool water content, stool samples were dried at $80^{\circ} \mathrm{C}$ for 24 hours and water content was calculated as follows: (wet weight - dry weight)/wet weight $(9,30)$.

Statistics. Experiments with 2 groups were analyzed using 2-tailed Student's $t$ test; for 3 or more groups, analysis was done with 1-way ANOVA and post hoc Newman-Keuls multiple comparisons test. For all analyses, $P$ values of less than 0.05 were considered statistically significant.

Study approval. Protocols were approved by the University of California, San Francisco, Institutional Animal Care and Use Committee.

\section{Author contributions}

OC and ASV conceived the study. OC, PMH, JATT, and AAR performed the experiments. OC, $\mathrm{PMH}$, and JATT analyzed the data. OC, PMH, and ASV wrote the paper.

\section{Acknowledgments}

This work was supported by grants from the NIH (DK072517, DK126070) and the Cystic Fibrosis Foundation (CIL21G0, VERKMA18G0). The authors thank Puay-Wah Phuan (University of California, San Francisco) and Marc O. Anderson (San Francisco State University) for advice on inhibitor chemistry.

Address correspondence to: Onur Cil, Department of Pediatrics, University of California, San Francisco, 513 Parnassus Avenue, HSE 1244, San Francisco, California 94143, USA. Phone: 415.476.2423; Email: onur.cil@ucsf.edu.

1. Ledoussal C, et al. Loss of the NHE2 $\mathrm{Na}(+) / \mathrm{H}(+)$ exchanger has no apparent effect on diarrheal state of NHE3-deficient mice. Am J Physiol Gastrointest Liver Physiol. 2005;281(6):G1385-G1396.

2. Mousavi TS, et al. An update on efficacy and safety considerations for the latest drugs used to treat irritable bowel syndrome. Expert Opin Drug Metab Toxicol. 2020;16(7):583-604.

3. Kato A, Romero MF. Regulation of electroneutral NaCl absorption by the small intestine. Annu Rev Physiol. 2011;73:261-281.

4. Simpson JE, et al. PAT-1 (Slc26a6) is the predominant apical membrane $\mathrm{Cl}-/ \mathrm{HCO} 3$ - exchanger in the upper villous epithelium of the murine duodenum. Am J Physiol Gastrointest Liver Physiol. 2007;292(4):G1079-G1088.

5. Wang Z, et al. Identification of an apical $\mathrm{Cl}(-) / \mathrm{HCO}(-)$ exchanger in the small intestine. Am J Physiol Gastrointest Liver Physiol. 2002;282(3):G573-G579.

6. Walker NM, et al. Down-regulated in adenoma $\mathrm{Cl} / \mathrm{HCO} 3$ exchanger couples with $\mathrm{Na} / \mathrm{H}$ exchanger 3 for $\mathrm{NaCl}$ absorption in murine small intestine. Gastroenterology. 2008;135(5):1645-1653.

7. Haggie PM, et al. SLC26A3 inhibitor identified in small molecule screen blocks colonic fluid absorption and reduces constipation. JCI Insight. 2018;3(14):121370.

8. Lee S, et al. 4,8-Dimethylcoumarin inhibitors of intestinal anion exchanger slc26a3 (downregulated in adenoma) for anti-absorptive therapy of constipation. J Med Chem. 2019;62(17):8330-8337.

9. Cil O, et al. CFTR activator increases intestinal fluid secretion and normalizes stool output in a mouse model of constipation. Cell Mol Gastroenterol Hepatol. 2016;2(3):317-327.

10. Cil O, et al. Small-molecule inhibitors of pendrin potentiate the diuretic action of furosemide. J Am Soc Nephrol. 2016;27(12):3706-3714.

11. Haggie PM, et al. Inhibitors of pendrin anion exchange identified in a small molecule screen increase airway surface liquid volume 
in cystic fibrosis. FASEB J. 2016;30(6):2187-2197.

12. Arvans D, et al. Activation of the PKA signaling pathway stimulates oxalate transport by human intestinal Caco2-BBE cells. Am J Physiol Cell Physiol. 2020;318(2):C372-C379.

13. Oak AA, et al. Repurposing calcium-sensing receptor agonist cinacalcet for treatment of CFTR-mediated secretory diarrheas. JCI Insight. 2021;6(4):e146823.

14. Radanovic T, et al. Regulation of intestinal phosphate transport. I. Segmental expression and adaptation to low-P(i) diet of the type IIb Na(+)-P(i) cotransporter in mouse small intestine. Am J Physiol Gastrointest Liver Physiol. 2005;288(3):G496-G500.

15. Kelly T, Buxbaum J. Gastrointestinal manifestations of cystic fibrosis. Dig Dis Sci. 2015;60(7):1903-1913.

16. Seidler U, et al. Sodium and chloride absorptive defects in the small intestine in Slc26a6 null mice. Pflugers Arch. 2008;455(4):757-766.

17. Xia W, et al. The distinct roles of anion transporters Slc26a3 (DRA) and Slc26a6 (PAT-1) in fluid and electrolyte absorption in the murine small intestine. Pflugers Arch. 2014;466(8):1541-1556.

18. Whittamore J, Hatch M. Loss of the anion exchanger DRA (Slc26a3), or PAT1 (Slc26a6), alters sulfate transport by the distal ileum and overall sulfate homeostasis. Am J Physiol Gastrointest Liver Physiol. 2017;313(3):G166-G179.

19. van der Doef HP, et al. Constipation in pediatric cystic fibrosis patients: an underestimated medical condition. J Cyst Fibros. 2010;9(1):59-63.

20. Knauf F, et al. Loss of cystic fibrosis transmembrane regulator impairs intestinal oxalate secretion. J Am Soc Nephrol. 2017;28(1):242-249.

21. Simpson JE, et al. Chloride conductance of CFTR facilitates basal Cl-/HCO3- exchange in the villous epithelium of intact murine duodenum. Am J Physiol Gastrointest Liver Physiol. 2005;288(6):G1241-G1251.

22. Sirish P, et al. Action potential shortening and impairment of cardiac function by ablation of Slc26a6. Circ Arrhythm Electrophysiol. 2017;10(10):e005267.

23. Jiang Z, et al. Calcium oxalate urolithiasis in mice lacking anion transporter Slc26a6. Nat Genet. 2006;38(4):474-478.

24. Monico CG, et al. Phenotypic and functional analysis of human SLC26A6 variants in patients with familial hyperoxaluria and calcium oxalate nephrolithiasis. Am J Kidney Dis. 2008;52(6):1096-1103.

25. Seidler U, Nikolovska K. Slc26 family of anion transporters in the gastrointestinal tract: expression, function, regulation, and role in disease. Compr Physiol. 2019;9(2):839-872.

26. Alper SL, Sharma AK. The SLC26 gene family of anion transporters and channels. Mol Aspects Med. 2013;34(2-3):494-515.

27. Karthikeyan C, et al. IND-2, a pyrimido[1",2":1,5]pyrazolo[3,4-b]quinoline derivative, circumvents multi-drug resistance and causes apoptosis in colon cancer cells. Bioorg Med Chem. 2015;23(3):602-11.

28. Cil $\mathrm{O}$, et al. Slowed gastric emptying and improved oral glucose tolerance produced by a nanomolar-potency inhibitor of calciumactivated chloride channel TMEM16A. FASEB J. 2019;33(10):11247-11257.

29. Cil O, et al. Benzopyrimido-pyrrolo-oxazine-dione CFTR inhibitor (R)-BPO-27 for antisecretory therapy of diarrheas caused by bacterial enterotoxins. FASEB J. 2016;31(2):751-760.

30. Cil O, et al. Phenylquinoxalinone CFTR activator as potential prosecretory therapy for constipation. Transl Res. 2017;182:14-26. 